\title{
Management of Early Menopause Using Traditional Persian Medicine: A Case Report
}

\author{
Nasim Behnoud ${ }^{1,2,3}{ }^{\circledR}$, Rosita Bahrami ${ }^{1,2,3}$, Gholamreza Kordafshari ${ }^{4}$, Farahnaz Farzaneh ${ }^{5}$, Hoorieh \\ Mohammadi Kenari ${ }^{2,3^{*} \mathbb{D}}$
}

\begin{abstract}
Interruption of the activity of ovary before the age of forty is called premature ovarian failure (POF) in which ovaries lose their follicular and hormonal functions. A decline in the number of ovules before the age of menopause is a physiologic phenomenon. In the present case report, treatment of a patient by POF was reported using traditional Persian medicine principles. In Persian medicine, each humor has its own temperament. A change in the temperament and quality or quantity of these humors causes the disease and therefore modifying the temperament is the milestone of treatment. The patient was a 39-year-old married woman with irregular menstruation cycles and finally, cessation of menstruation. In this period, the patient had vaginal dryness, severe dyspareunia, gastritis, and parasomnia. At first, some instructions were ordered to change her lifestyle, for example, a change in food intakes, enough sleep, and exercise. In addition, several medications were ordered including the digestive system electuary (Majoun Jahaz Hazemeh), Eyaraj Fighara capsule and powder containing Glycyrrhiza glabra roots, Foeniculum vulgare and white sugar, oil massage of the abdomen and flanks, and cupping of the uterus. At the end of treatment, complete rehabilitation was achieved and menstruation irregularity, gastritis, and parasomnia were resolved. Based on the results, if a patient follows the rules and has a healthy lifestyle, inappropriate humor is unable to appear, the healing can be stable, and this disease or similar melancholic disorders fail to occur.
\end{abstract}

Keywords: Premature ovarian failure, Menopause, Persian medicine

\section{Introduction}

During the past few decades, the rate of fertility has dramatically reduced in all the countries worldwide. However, the magnitude of such a decline varies in various countries (1). Early menopause and abnormal early infertility can affect self-efficacy and self-esteem (2). Premature ovarian failure (POF) is the disruption of the activity of ovaries before the age of forty. This appears when ovaries lose their follicular and hormonal functions. In fact, a decrease in the number of ovaries follicles before the age of menopause is considered physiologic (3). According to the definition of the World Health Organization (WHO), menopause is defined as stoppage of menstruation bleeding due to deactivation of ovarian follicles accompanied by twelve months of bleeding stoppage or amenorrhea during the age of 45-55 (4). It seems that POF occurs when the follicles number is decreased by autoimmune damage accompanied by genetic predisposing factors. This happens in $1 \%$ of the women younger than 40 years and $0.1 \%$ before the age of thirty (5). Based on the results of the studies performed in Iran, the age range of menopause is 46 to 52 years
(6). This phenomenon affects women' health status and quality of life by hormonal changes (7). It is thought that chronic diseases including cardiovascular disorders and several malignancies like endometrium and breast cancers are due to the activity of ovary function (8). In addition, premature menopause can increase the risk of cardiovascular diseases and bone loss (9). Menopausal symptoms were highlighted as bone disease, hot flash, parasomnia, anxiety, skin shrinkage, and irritability and different studies represented an association between these symptoms and the age of menopause (10). Therefore, menopause is of great importance due to its effects on health status. Hormone replacement therapy (HRT) is an acceptable treatment for POF of the patients, which maintains the natural cycle. However, it has its own side effects such that some physicians and patients prefer complementary and alternative medicine (2). Several studies were conducted regarding using alternative therapies in the treatment of different developer factors of infertility (11-14). In traditional Persian medicine, all creatures are composed of 4 elements of water, fire, air, and soil which include the four temperament theories 
(Blood, Yellow bile, Phlegm, and Black bile). Each humor has some temperament (Mizaj). For instance, blood is hot and watery, Yellow bile is hot and dry, phlegm is cold and moist, and finally, black bile is cold and dry. From the perspective of Persian medicine, an alteration in temperament, as well as quality and quantity of these humors is the reason for the emergence of the diseases and thus temperament modification is regarded as the milestone of treatment in this respect. In a healthy individual, quadruple humors are in an equilibrium (1517) in which the stability is achieved by performing the six principles of health (Sette Zaroorieh) including weather health, food, normal body activity, sleep, keeping and excreting chemicals from the body, and mental health. Medicine and supporting activities such as cupping and bloodletting and phlebotomy can be used if necessary (18, 19). The present case report is related to the treatment of POF using traditional Persian medicine aiming at puberty induction, life expectancy, and implications for relatives of the case under investigation.

\section{Case Presentation}

The patient was a 39-year-old female and married having two children aged 16 and 24 years. Further, she was a housewife who lived in Tehran. She had a weight of 64 $\mathrm{kg}$ and a height of $155 \mathrm{~cm}$. Furthermore, she reported irregular menstruation from 2 years ago. At first, she had 2 times of metrorrhagia and clots discharge from the vagina. Then, she had menstruation after an interval of 2-3 months and using progesterone ampulla and finally, complete amenorrhea occurred. The patient was referred to a gynecologist diagnosed with early menopause while receiving no medications. In this period, the patient experienced vaginal dryness, severe dyspareunia, and low libido. Physical examination revealed cervicitis. Moreover, inflammation in pop smear was reported which was burned with cutter once and antibiotic therapy was performed followed by antibiotic therapy. Both breasts had hazy pains while no abnormality was observed in ultrasonography or mammography investigations. Additionally, the patient complained about dyspepsia, heartburn, and stomachache. She underwent upper gastrointestinal (GI) endoscopy showing gastritis. In addition, nausea and vomiting (twice a week) and epigastric pain normally occurred in the morning. Further, she complained about having a headache and vomiting twice a week. Furthermore, she reported obsessive thoughts, parasomnia, nightmares, darkness in face skin, and itching. Based on history taking, she was living in an apartment in Tehran consuming meal three times per day. She had many limitations in food and beverage consumption like some fruits such as cucumber, squash, melons, as well as milk and yogurt. She slept at 2 AM until 7 to 8 and felt a loss of energy in the morning and typically exhausted. She did not exercise. Her defecation, urination, and sweating were normal. Moreover, her menstruation typically lasted for 7 days during which she had severe vaginal bleeding with clots at first three days and spotting lasting for 7 days. Her physical examination revealed a normal vital singe with no positive symptom. Finally, the first lab examinations included $\mathrm{FSH}=1.45$ and $\mathrm{LH}=6.37$. Other hormonal laboratories obtained normal findings.

Investigating her family records it was revealed that her mother, aunt, and grandmother underwent a hysterectomy at the age of 40 years due to abnormal uterine bleeding and fibroma.

Therefore, therapeutic methods of traditional Persian medicine (i.e., modification in lifestyle, food intakes, sleep and exercise, and medications) to treat this patient.

On the first visit, after taking a complete history including diet, sleeping, and daily physical activity, some tips were provided to improve her lifestyle. She was asked not to eat different foods together, not to overeat, or eating extremely fast while eating a three-course meal on time, avoid drinking beverages, yogurt, or salad with the main food and only consuming olive with her food. She was allowed to have salad, fruits, syrups, and yogurt as a snack and after an hour from the main servings. Additionally, she was recommended to do ten to fifteen minutes of gentle exercise including jogging or walking 2-3 times a week. Eventually, on time sleeping and waking, that is going to bed before $11 \mathrm{PM}$ and waking before $6 \mathrm{AM}$ while not having a daily nap except for a maximum of 30 minutes.

In addition, the following medicines or remedies were prescribed:

- A powder containing Glycyrrhiza glabra, Foeniculum vulgare, and white sugar, one teaspoon before each food serving;

- One teaspoon of a mixture of digestive system electuary (Majoun Jahaz Hazemeh) based on rooster gizzard half an hour after the food;

- An Eyaraj Fighara capsule each afternoon;

- Massage of abdomen and flanks with a mixture of Nigella Sativa, Matricaria chamomilla, and Costus oil 3-4 times a week;

- hypogastric cupping every other day.

Forty days later on the second visit, by consuming and performing the above-mentioned medicines and remedies, she had one time of menstruation which lasted for 5 days with less dysmenorrhea. Heartburn was healed. Therefore, she was asked to continue the aforementioned items and add cases including applying Rosa damascena oil and honey in the vagina using a special applicator, using a sit bath on the boiled flower and leaves of Althaea officinalis, Malva neglecta, Linum usitatissimum, and scheduling the next visit within one month. However, she came after 5 months for the third visit reporting that she did the exercises, had a proper food regimen and a regular sleep cycle and that her stomachache relieved. She further explained that she had somehow irregular menstruation during these 5 months. Vaginal discharge and infection healed and no dryness or dyspareunia remained. 
Ultimately, she referred to a gynecologist and underwent pop smear in which vaginal inflammation was found to be healed. At this stage, medicines tapered off and levels of FSH and LH evaluated.

One month later at the fourth visit, she had no complaint about heartburn, dyspareunia, or itching. Vaginal bleeding was normal and libido returned. Furthermore, FSH and LH levels were 19.11 and 8.13, respectively. The patient was advised to follow lifestyle modifications and return in case of any problem. Six months later on a telephone call follow-up, the patient reported having a regular mensuration while not complaining about digestive or sexual problems.

\section{Discussion}

In modern medicine, the etiology of amenorrhea includes structural or functional dysfunction in sexual organs like the ovarian failure, lesions of the hypothalamichypophyseal axis, or abnormal secretion of the gonadotropin-releasing hormone from the hypothalamus and reproductive systems.

Conversely, in traditional Persian medicine, the etiology of amenorrhea is divided into uterus etiology including the obstruction or blockage in the uterus due to dystemperament or inflammations, and the like, as well as common etiologies such as occasional dystemperament in the body or any disease in other organs which may disrupt mensuration $(15,20,21)$. Several studies were found to use traditional and herbal methods to treat early menopause $(2,22-24)$

The patient of the current study reported having cold and dry dystemperament (suolmizaj) based on traditional medicine rules, which caused complaints about vaginal dryness, dyspareunia, obsessions thoughts, parasomnia, nightmare, the darkness of skin color, and dryness and itching of the skin. Digestive disorders of the patient were due to cold and dry dystemperament in the stomach leading to dyspepsia through which the elements fail to be properly absorbed and therefore are unable to reach the other parts properly.

Based on the principles of traditional Persian medicine, it was attempted to treat the disease basically. The first step to treat this condition was to modify the lifestyle and obey the 6 necessary principles of life (Sette Zaroorieh), which was explained in (25), followed by treating the main reason of cold and dry dystemperament probably caused by digestive disorders. Modifying food consumption, as well as avoiding eating cold and dry foods, overeating, and consuming irregular food may improve the function of the digestive system leading to proper humor. Moreover, eating olive can improve function and digestion of the GI system $(26,27)$. Therefore, she was advised to eat olive with her food.

Daily exercise in short periods gives energy and increases basic body metabolism and body temperature, as a result, natural intelligence of the body is fortified and can confront the disease related to inappropriate humors in the body (27). However, long exercise was not advised to this patient since it can increase body dryness. Conversely, having enough sleep and regulating sleep time are effective in decreasing the mental problems and treating cold and dry dystemperament since parasomnia may lead to inappropriate dryness of the body.

A powder containing G. glabra root, F. vulgare, and white sugar was given to the patient which, based on traditional Persian medicine, can open the body ducts, remove dyspepsia, and improve the skin color and face. Each part of this mixture was helpful for the patient. For example, G. glabra root could heal the abnormal humors, fortify the nervous system, remove dyspepsia, and was emmenagogue. Additionally, F. vulgare, as an emmenagogue herb, was found to fortify the stomach, excrete thin or dense moisture of the stomach, and relieve nausea. In addition, the effects of $F$. vulgare on treating the amenorrhea was reported in some studies $(27,28)$ while several other studies emphasized the effect of G. glabra root on regulating the estrogen and treating polycystic ovary $(29,30)$.

The next medicine was related to digestive system electuary or gizzard which fortifies stomach and heart and heals dyspepsia. Traditional medicine practitioners believed that gases and harmful excretion are produced by partial digestion if the digestive system fails to act properly and move to upper portions of the body due to their light weight and thus cause mental and brain complications differing based on the type of the excreted gases $(15,31$, 32) The patient who was examined in the present study had symptoms which were due to the presence of black bile in the brain. Accordingly, electuary (Majoun Jahaz Hazemeh) was given to the patient to fortify her digestive system 30 minutes after food consumption in order to improve the GI system, achieve appropriate humor, and prevent the ascending of the gases. Further, Eyaraj Fighara capsule, which is a combination of Aloe vera, Valeriana officinalis, Zingiber officinale, Pistacia lentiscus, Cinnamomum zeylanicum, and Crocus sativus was given to the patient in order to remove previous gases gathered in her stomach and brain. This capsule cleans the brain, stomach, and uterus and absorbs and separating the secretion of the head by guts (33). Each of the abovementioned parts has its own properties for fortifying and cleaning the other organs, especially strengthening the intelligent nature of the body (34).

In this patient, gentle massage of the belly and flanks by a mixture of oils such as Nigella sativa, C. zeylanicum, and Costus which warms the inner organs, especially the stomach and uterus and fortifies them. Furthermore, oils like Chamomile and Costus are dissolvent and antiinflammatory or dyspepsia and thus remove coldness of the organs and open the closed ducts blocked due to the coldness. Many studies confirmed the effect of oil massage on the GI system, especially regarding the effect 
of Chamomile on improving the bowel movement (35). Moreover, cupping on belly and areas around the uterus was performed to achieve in this regard since cupping causes more absorption of blood flow in the area and warms the organ and drains the aggregated harmful excretions from the uterus to the skin in order to remove them $(36,37)$. Additionally, improving the function of the GI system resulted in resolving the uterus problems to some extent. Traditional medicine scientists believed that stomach and uterus have strong innervation and closely collaborate with the brain. Therefore, any medicine with an effect on the nervous system has an effect on the abovementioned organs, which had some dysfunction in the patient of the current study.

Finally, the researchers' expectation was met during the second visit. Therefore, a mixture of Rosa damascene oil and honey was transvaginally applied for better healing of the uterus and treating menopause or dystemperament of the uterus which was considered the frequent complaint of the patient. This mixture improves uterus function and excretes bad secretions of the uterus. Different studies approved the effect of $R$. damascena and honey on the treatment of dermatologic diseases (38) and vaginal problems (39-41). In addition, sitting in a mixture of Althaea officinalis, Malva neglecta, and Linum usitatissimum was prescribed, which helps to remove the inflammation of uterus and its dystemperament. Further, herbal medicines like A. officinalis and Malva neglect the excretion of harmful secretions and do not allow the re-gathering of such secretions and bad humors in any organs. This property helps the excretion of previously collected secretions, prevents absorption of bad resources, and increases the stability of treatment. In recent studies, anti-candida effects and anti-inflammatory properties of Malva neglecta and A. officinalis family were confirmed by intravaginal use, respectively (31-33, 42, 43). Accordingly, cervicitis and chronic vaginal infection were cured when the patient consumed this medication. Considering the process of disease and its healing based on our expectations, atrabilious substances, as well as cold and dry dystemperament properly healed. Furthermore, the reoccurrence of atrabilious humor was prevented given the necessity of obeying the 6 vital principles (Sette Zaroorieh) and modifying the lifestyle. Moreover, inappropriate humor may not occur in the body while the patient performs the orders and follows the correct lifestyle. Finally, the treatment lasts, whereas the disease is unable to relapse or even similar diseases fail to reoccur.

\section{Conclusions}

Generally, the present case study was an example of treating a patient by the principles of traditional Persian medicine. It seems that menopause can be postponed by obeying the health principles mentioned in Persian medicine. However, other studies in this respect are subject to further investigations. Additionally, establishing a close collaboration between modern and traditional medicine is recommended in this field in order to perfectly treat such disorders. The incidence of such problems may reduce by educating these principles and women can have more years of fertility. Eventually, in the case of any dysfunction of the ovary, the best treatment can be administered based on traditional Persian medicine.

\section{Conflict of Interests}

Authors declare that they have no conflict of interests.

\section{Ethical Issues}

Written informed consent was obtained from the patient for publication of this case report.

\section{Financial Support}

None declared.

\section{Acknowledgments}

The authors wish to thank the patient who followed the prescriptions and allowed us to report the process of her treatment.

\section{References}

1. Filoso V, Papagni E. Fertility choice and financial development. Eur J Polit Econ. 2015;37:160-177. doi:10.1016/j.ejpoleco.2014.11.004

2. Lin J, Li XL, Song H, et al. A general description for Chinese medicine in treating premature ovarian failure. Chin J Integr Med. 2017;23(2):91-97. doi:10.1007/s11655-0162642-7

3. Jankowska K. Premature ovarian failure. Prz Menopauzalny. 2017;16(2):51-56. doi:10.5114/pm.2017.68592

4. Kapur P, Sinha B, Pereira BM. Measuring climacteric symptoms and age at natural menopause in an Indian population using the Greene Climacteric Scale. Menopause. 2009;16(2):378-384. doi:10.1097/gme.0b013e31818a2be9

5. Anasti JN. Premature ovarian failure: an update. Fertil Steril. 1998;70(1):1-15. doi:10.1016/S0015-0282(98)000995

6. Sadat-Hashemi SM, Ghorbani R, Kalalian H, Askari Majdabadi H, Kaveei B, Khalajabadi Farahani F. Parametric Estimation of Age Patterns at Natural Menopause in Shahrood; Semnan Province, Iran, 2007. The Iranian Journal of Obstetrics, Gynecology and Infertility. 2009;12(3):29-35. doi:10.22038/ijogi.2009.5886

7. Vogelvang TE, van der Mooren MJ, Mijatovic V, Kenemans P. Emerging selective estrogen receptor modulators: special focus on effects on coronary heart disease in postmenopausal women. Drugs. 2006;66(2):191-221. doi:10.2165/00003495-200666020-00005

8. Cagnacci A, Cannoletta M, Caretto S, Zanin R, Xholli A, Volpe A. Increased cortisol level: a possible link between climacteric symptoms and cardiovascular risk factors. Menopause. 2011;18(3):273-278. doi:10.1097/ gme.0b013e3181f31947

9. Pouresmaeili F, Fazeli Z. Premature ovarian failure: a critical condition in the reproductive potential with various genetic 
causes. Int J Fertil Steril. 2014;8(1):1-12.

10. Ayranci U, Orsal O, Orsal O, Arslan G, Emeksiz DF. Menopause status and attitudes in a Turkish midlife female population: an epidemiological study. BMC Womens Health. 2010;10:1. doi:10.1186/1472-6874-10-1

11. Soltani M, Moghimian M, Abtahi H, Shokoohi M. The protective effect of Matricaria chamomilla extract on histological damage and oxidative stress induced by Torsion/Detorsion in adult rat ovary. International Journal of Women's Health and Reproduction Sciences. 2017;5(3):187-92. doi:10.15296/ijwhr.2017.34

12. Chen Y, Fang Y, Yang J, Wang F, Wang Y, Yang L. Effect of acupuncture on premature ovarian failure: a pilot study. Evid Based Complement Alternat Med. 2014;2014:718675. doi:10.1155/2014/718675

13. Soltani M, Moghimian M, Abtahi-Eivari SH, Shoorei H, Khaki A, Shokoohi M. Protective Effects of Matricaria chamomilla Extract on Torsion/ Detorsion-Induced Tissue Damage and Oxidative Stress in Adult Rat Testis. Int J Fertil Steril. 2018;12(3):242-248. doi:10.22074/ijfs.2018.5324

14. Shoorei H, Khaki A, Ainehchi N, et al. Effects of Matricaria chamomilla Extract on Growth and Maturation of Isolated Mouse Ovarian Follicles in a Three-dimensional Culture System. Chin Med J (Engl). 2018;131(2):218-225. doi:10.4103/0366-6999.222324

15. Ibn-e-Sina. Al-Qanun Fit-tib (vol 3) [The Canon of Medicine]. Beirut; 2005.

16. Aghili Alavi Khorasani MH. Makhzan ol Advieh. Tehran:Intisharat va Amoozesh Enghelab Islami Press; 1992.

17. Gorji A, Khaleghi Ghadiri M. History of headache in medieval Persian medicine. Lancet Neurol. 2002;1(8):510515. doi:10.1016/S1474-4422(02)00226-0

18. Larijani B, Esfahani MM, Moghimi M, et al. Prevention and Treatment of Flatulence From a Traditional Persian Medicine Perspective. Iran Red Crescent Med J. 2016;18(4):e23664. doi:10.5812/ircmj.23664

19. Kordafshari G, Ardakani MRS, Keshavarz M, et al. The Role of Phlebotomy ( Fasd) and Wet Cupping ( Hijamat) to Manage Dizziness and Vertigo From the Viewpoint of Persian Medicine. J Evid Based Complementary Altern Med. 2017;22(3):369-373. doi:10.1177/2156587216672757

20. Mokaberinejad R, Zafarghandi N. Etiology and semiology of amenorrhea in the traditional Iranian medicine. Journal of Islamic and Iranian Traditional Medicine. 2012;3(1):1930 .

21. Nazem Jahan MA. Eksir-e-Azam. Tehran: Institute for Islamic and Complementary Medicine; 2009:704-753. [Persian].

22. Wu Y, Chen LT, Qu F, Sheikh IS, Wu YT. Chinese Herbal Medicine for premature ovarian failure: a systematic review and meta-analysis. Eur J Integr Med. 2014;6(3):382-391. doi:10.1016/j.eujim.2014.01.008

23. Edmonds SE, Montgomery JC. Reversible ovarian failure induced by a Chinese herbal medicine: lei gong teng. BJOG. 2003;110(1):77-78. doi:10.1046/j.1471-0528.2003.t01-102039.x

24. Pitkin J. Alternative and complementary therapies for the menopause. Menopause Int. 2012;18(1):20-27. doi:10.1258/ mi.2012.012001
25. Mohammadi Kenari H, Kordafshari G, Kamalinejad M, Aghighi S, Moghimi M. Treatment of Hospital Acquired Pneumonia (HAP) with Persian medicine (PM): A Case Report. Tradit Integr Med. 2018;3(2):69-7.

26. Aghili Khorasani MH. [Makhzan ul-Advia]. Tehran: Bavardaran Publication; 2001:122-123. [Persian].

27. Kordafshari G, Mohamadi Kenari H, Nazem E, et al. The Role of Nature (Tabiat) in Persian Medicine. Tradit Integr Med. 2017;2(4):177-181.

28. Bhatia H, Pal Sharma Y, Manhas RK, Kumar K. Traditional phytoremedies for the treatment of menstrual disorders in district Udhampur, J\&K, India. J Ethnopharmacol. 2015;160:202-210. doi:10.1016/j.jep.2014.11.041

29. Moini Jazani A, Hamdi K, Tansaz M, et al. Herbal Medicine for Oligomenorrhea and Amenorrhea: A Systematic Review of Ancient and Conventional Medicine. Biomed Res Int. 2018;2018:3052768. doi:10.1155/2018/3052768

30. Arentz S, Abbott JA, Smith CA, Bensoussan A. Herbal medicine for the management of polycystic ovary syndrome (PCOS) and associated oligo/amenorrhoea and hyperandrogenism; a review of the laboratory evidence for effects with corroborative clinical findings. BMC Complement Altern Med. 2014;14:511. doi:10.1186/14726882-14-511

31. Zhang JJ, Han IK, Zhang L, Crain W. Hazardous chemicals in synthetic turf materials and their bioaccessibility in digestive fluids. J Expo Sci Environ Epidemiol. 2008;18(6):600-607. doi:10.1038/jes.2008.55

32. Cole LA, Sasaki Y, Muller CY. Normal production of human chorionic gonadotropin in menopause. N Engl J Med. 2007;356(11):1184-1186. doi:10.1056/NEJMc066500

33. Khorasani A. Gharabadian-e-Kabir. Tehran: Mahmoudi Publication; 2000.

34. Kordafshari G, Kenari HM, Esfahani MM, et al. Nutritional aspects to prevent heart diseases in traditional Persian medicine. J Evid Based Complementary Altern Med. 2015;20(1):57-64. doi:10.1177/2156587214553939

35. Khadem E, Shirazi M, Janani L, Rahimi R, Amiri P, Ghorat F. Effect of Topical Chamomile Oil on Postoperative Bowel Activity after Cesarean Section: A Randomized Controlled Trial. J Res Pharm Pract. 2018;7(3):128-135. doi:10.4103/ jrpp.JRPP_17_103

36. Inanmdar W, Sultana A, Mubeen U, Rahman K. Clinical efficacy of Trigonella foenum graecum (Fenugreek) and dry cupping therapy on intensity of pain in patients with primary dysmenorrhea. Chin J Integr Med. 2016. doi:10.1007/s11655-016-2259-x

37. El Sayed SM, Al-quliti AS, Mahmoud HS, et al. Therapeutic benefits of Al-hijamah: in light of modern medicine and prophetic medicine. Am J Med Biol Res. 2014;2(2):46-71. doi:10.12691/ajmbr-2-2-3

38. Farnia V, Tatari F, Alikhani M, et al. Rosa Damascena oil improved sexual function and testosterone in male patients with opium use disorder under methadone maintenance therapy-results from a double-blind, randomized, placebo-controlled clinical trial. Drug Alcohol Depend. 2017;176:117-125. doi:10.1016/j.drugalcdep.2017.02.008

39. Nabimeybodi R, Meyari A, Vahiddastjerdi M, et al. The Effect of Flixweed-Honey Vaginal Product on Cervicitis: a Clinical Trial. Research Journal of Pharmacognosy. 
2018;5(2):41-49. doi:10.22127/rjp.2018.58506

40. Banaeian S, Sereshti M, Rafieian M, Farahbod F, Kheiri S. Comparison of vaginal ointment of honey and clotrimazole for treatment of vulvovaginal candidiasis: A random clinical trial. J Mycol Med. 2017;27(4):494-500. doi:10.1016/j. mycmed.2017.07.003

41. Darvishi M, Jahdi F, Hamzegardeshi Z, Goodarzi S, Vahedi $\mathrm{M}$. The Comparison of vaginal cream of mixing yogurt, honey and clotrimazole on symptoms of vaginal candidiasis. Glob J Health Sci. 2015;7(6):108-116. doi:10.5539/gjhs. v7n6p 108

42. Ogenler O, Un I, Uzel I. Medical plants used for treatment of gynecological disorders in Ottomans in the 15th century. J Complement Med Res. 2018;7(2):171-177. doi:10.5455/ jcmr.20180119120939

43. Soliman S, Alnajdy D, El-Keblawy AA, Mosa KA, Khoder G, Noreddin AM. Plants' Natural Products as Alternative Promising Anti-Candida Drugs. Pharmacogn Rev. 2017;11(22):104-122. doi:10.4103/phrev.phrev_8_17

(c) 2019 The Author (s); This is an open-access article distributed under the terms of the Creative Commons Attribution License (http://creativecommons.org/licenses/by/4.0), which permits unrestricted use, distribution, and reproduction in any medium, provided the original work is properly cited. 